\title{
FINITE-DIMENSIONAL BANACH SPACES WITH A.E. DIFFERENTIABLE METRIC PROJECTION
}

\author{
THEAGENIS ABATZOGLOU
}

\begin{abstract}
We prove that in a finite-dimensional Banach space, where the Hessian of the norm square is positive definite on the whole space, except possibly at 0 , the metric projection onto any closed set is differentiable a.e.
\end{abstract}

1. Introduction. Asplund in [2] proved that in any Euclidean space the metric projection onto a closed set is differentiable a.e. In the last paragraph of the same paper he asks whether this behaviour of the metric projection can be generalized to other norms.

We expand his approach and generalize his result to spaces where the Hessian of the square of the norm is positive definite in the whole space, except at the origin where the Hessian may not exist.

2. Definitions. Let $B$ be a finite-dimensional Banach space and $M$ a closed subset of $B$.

The metric projection $P$ of $B$ onto $M$ is a map, possibly multivalued, defined by

$$
\inf _{m \in M}\|x-m\|=\|x-P(x)\| .
$$

3. Differentiability of the metric projection. We prove a few lemmas to establish some preparatory and useful properties of the metric projection.

LEMma 1. Let $F(x)=\|x-P(x)\|$. Then $F$ is Lipschitz continuous and therefore Fréchet differentiable a.e. on $B$.

Proof. $F(y)-F(x)=\|y-P(y)\|-\|x-P(x)\|$ and without loss of generality assume $F(y)-F(x) \geqslant 0$. Then

$$
\begin{aligned}
|F(y)-F(x)| & =|\|y-P(y)\|-\|x-P(x)\|| \\
& \leqslant|\|y-P(x)\|-\|x-P(x)\||<\|y-x\| .
\end{aligned}
$$

The lemma now follows from the classical theorem of Rademacher and Stepanoff $[4$, p. 216].

LemMA 2. If $F$ is Fréchet differentiable at $x=P(x)$ then $\nabla\|x-P(x)\|=0$ at $x=P(x)$.

Proof. For any $y \in B$ consider

$$
\frac{\|x+t y-P(x+t y)\|-\|x-P(x)\|}{t}=\frac{\|x+t y-P(x+t y)\|}{t} .
$$

Received by the editors December 14, 1978.

AMS (MOS) subject classifications (1970). Primary 41A50.

(c) 1980 American Mathematical Society 0002-9939/80/0000-0158/\$02.25 
Since $\|x+t y-P(x+t y)\| \geq 0$ we get

$$
0=\lim _{t \rightarrow 0} \frac{F(x+t y)-F(x)}{t}=\nabla\|x-P(x)\|(y) \text { for all } y \in B .
$$

Therefore $\nabla\|x-P(x)\|=0$.

LeMma 3. If $F$ is Fréchet differentiable at $x=P(x)$ then $P^{\prime}(x)=I$, the identity map.

Proof. By Lemma 2, $\nabla\|x-P(x)\|=0$ at $x=P(x)$, i.e.,

$$
\lim _{t \rightarrow 0}\|x+t y-P(x+t y)\| / t=0 \text { for all } y \in B,
$$

therefore

$$
x+t y-P(x+t y)=o(|t|)
$$

and

$$
\left.\frac{d}{d t} P(x+t y)\right|_{t=0}=y .
$$

Theorem 1. $P$ is differentiable a.e. on $B$ if and only if $P$ is differentiable a.e. on $M^{C}$ (the complement of $M$ in $B$ ).

Proof. Follows easily from Lemmas 1 and 3.

From now on we will concentrate on the function $F(x)=\|x-P(x)\|$ on $M^{C}$ and prove that $F^{2}(x)$ is twice differentiable a.e. and from this it will follow that $P$ is differentiable a.e. on $M^{C}$.

LeMMA 4. If $F$ is Fréchet differentiable at $x$ then $F^{\prime}(x)=(x-P(x))^{*}$, where $(x-P(x))^{*}$ is the support functional to $x-P(x)$.

Proof. Let $y=(x-P(x)) /\|x-P(x)\|$. When $t<0$ and sufficiently close to 0 it is clear that $P(x+t y)=P(x)$. We then have

$$
\frac{F(x+t y)-F(x)}{t}=\frac{\|x+t y-P(x)\|-\|x-P(x)\|}{t}
$$

and

$$
\begin{aligned}
F^{\prime}(x)(y) & =\lim _{t \rightarrow 0^{-}} \frac{\|x+t y-P(x)\|-\|x-P(x)\|}{t}=(x-P(x))^{*}(y) \\
& =(x-P(x))^{*} \frac{x-P(x)}{\|x-P(x)\|}=1 .
\end{aligned}
$$

Now $F^{\prime}(x)$ is a linear functional of norm at most 1 because, by Lemma 1 , $|F(y)-F(x)| \leqslant\|y-x\|$. This means that $F^{\prime}(x)$ is a support functional to $x-$ $P(x)$.

Since the norm in $B$ is differentiable, support functionals are unique so that $F^{\prime}(x)=(x-P(x))^{*}$.

Corollary 1.P is uniquely defined a.e. on $B$. 
From now on we will assume that $G(x)=2\|x\|^{2}$ is twice Fréchet differentiable for all $x \neq 0$ and that $G^{\prime \prime}(x)$ is positive definite for all $x \neq 0$ in $B$. This has the following consequence.

LeMMA 5. $G^{\prime \prime}$ satisfies the following inequality for all $x, y$ in $B, x \neq 0$ and some $m, M>0$ :

$$
m\|y\|^{2} \leqslant G^{\prime \prime}(x)(y)^{(2)}<M\|y\|^{2} .
$$

Proof. $G$ is homogenous of degree 2, therefore $G^{\prime \prime}$ is homogenous of degree 0 . For any $x, y$ in $B$ with $\|x\|=1,\|y\| \leqslant 1$ by the compactness of the unit ball in $B$ and the continuity of $G^{\prime \prime}$ we obtain

$$
0<m=\inf _{\substack{\|x\|=1 \\\|y\|=1}} G^{\prime \prime}(x)(y)^{(2)},
$$

and

$$
\sup _{\substack{\|x\|=1 \\\|y\|=1}} G^{\prime \prime}(x)(y)^{(2)}=M<\infty .
$$

Now since $G^{\prime \prime}$ is homogenous of degree 0 it follows that for every $x, y$ in $B, x \neq 0$, we have

$$
m\|y\|^{2}<G^{\prime \prime}(x)(y)^{(2)}<M\|y\|^{2} .
$$

LEMMA 6. For any $x, y, z$ in $B$ we have the inequality

$$
2\|x+z\|^{2}+2\|y+z\|^{2}-\|x+y+2 z\|^{2}<\frac{M}{m}\left(2\|x\|^{2}+2\|y\|^{2}-\|x+y\|^{2}\right) .
$$

Proof. Let $H(t)=2\|t x+(1-t) y\|^{2}$. By Taylor's theorem with remainder we get

$$
H(1)+H(0)-2 H(1 / 2)=\int_{0}^{1 / 2} t H^{\prime \prime}(t) d t+\int_{1 / 2}^{1}(1-t) H^{\prime \prime}(t) d t
$$

Now $H^{\prime \prime}(t)=G^{\prime \prime}(y+t(x-y))(x-y)^{(2)}$ and

$$
H(1)+H(0)-2 H(1 / 2)=2\|x\|^{2}+2\|y\|^{2}-\|x+y\|^{2}
$$

so that if we use Lemma 5 on (1) we obtain

$$
\frac{1}{4} m\|x-y\|^{2} \leqslant 2\|x\|^{2}+2\|y\|^{2}-\|x+y\|^{2}<\frac{1}{4} M\|x-y\|^{2} .
$$

Observe that if $t x+(1-t) y=0$ for some $t$, the above inequalities still hold because $m<4$ and $M \geqslant 4$. Lemma 6 follows easily from this last inequality.

Theorem 2. Let $M$ be a closed set in $B$. Then $P$ is Fréchet differentiable a.e. on $B$.

Proof. Consider the function $K(x)=C\|x\|^{2}-\left\|x-P(x)^{2}\right\|$ where $C=M / m$. $K$ is clearly continuous, so to prove $K$ is convex it is enough to show $K$ is midpoint convex. The condition for this is

$$
\begin{aligned}
C\|x+y\|^{2}- & \|x+y-P(x+y)\|^{2} \\
& <\frac{1}{2}\left[C\|2 x\|^{2}-\|2 x-P(2 x)\|^{2}+C\|2 y\|^{2}-\|2 y-P(2 y)\|^{2}\right] .
\end{aligned}
$$


If we let $P(x+y)=-2 z$ it is enough to show

$$
2\|x+z\|^{2}+2\|y+z\|^{2}-\|x+y+2 z\|^{2}<C\left(2\|x\|^{2}+2\|y\|^{2}-\|x+y\|^{2}\right) .
$$

However this last inequality is true by Lemma 6. By Alexandrov's Theorem (see [1] or [3, p. 24]), $K$ has a second differential a.e. on $B$. This means that for almost all $x$ in $B$ we have

$$
K(y)=K(x)+K^{\prime}(x)(y-x)+\frac{1}{2} K^{\prime \prime}(x)(y-x)^{(2)}+o\left(\|y-x\|^{2}\right) .
$$

In particular this implies that if $\underline{K}^{\prime}(y)$ is a subdifferential of $K$ at $y$ then

$$
\underline{K}^{\prime}(y)-K^{\prime}(x)=K^{\prime \prime}(x)(y-x)+o(\|y-x\|) .
$$

A proof of (2) was cordially communicated to us by Professor Simon Fitzpatrick and it goes as follows.

From equation (1) we have, for every $\varepsilon>0$, a $\delta>0$ such that if $\|y-x\|<\delta$ then

$$
\left|K(y)-K(x)-\left\langle K^{\prime}(x), y-x\right\rangle-\frac{1}{2}\left\langle K^{\prime \prime}(y-x), y-x\right\rangle\right|\left\langle\varepsilon\|y-x\|^{2} .\right.
$$

Take $z, w$ in $B$ with $\|z\|=\|w\|=1$ and let $0<|t|<\delta / 2$ and $\alpha=\sqrt{\varepsilon}|t|$. Suppose $\underline{K}^{\prime}(x+t w)$ is a subdifferential of $K$ at $x+t w$. Then

$$
\left\langle\underline{K}^{\prime}(x+t w), \alpha z\right\rangle\langle K(x+t w+\alpha z)-K(x+t w)
$$

and by (3) (assuming $\varepsilon<1$ ),

$$
\begin{aligned}
K(x+t w+\alpha z)<\varepsilon 4|t|^{2}+K(x)+\left\langle K^{\prime}(x), t w+\alpha z\right\rangle \\
+\frac{1}{2}\left\langle K^{\prime \prime}(x)(t w+\alpha z), t w+\alpha z\right\rangle
\end{aligned}
$$

and

$$
K(x+t w) \geqslant-\varepsilon|t|^{2}+K(x)+\left\langle K^{\prime}(x), t w\right\rangle+\frac{1}{2}\left\langle K^{\prime \prime}(t w), t w\right\rangle .
$$

Combining the last three inequalities we obtain

$$
\begin{aligned}
\left\langle\underline{K}^{\prime}(x+t w), \alpha z\right\rangle\langle & 5 \varepsilon|t|^{2}+\left\langle K^{\prime}(x), \alpha z\right\rangle+\frac{1}{2}\left\langle K^{\prime \prime}(x)(t w), \alpha z\right\rangle \\
& +\frac{1}{2}\left\langle K^{\prime \prime}(x)(\alpha z), t w\right\rangle+\frac{1}{2}\left\langle K^{\prime \prime}(x)(\alpha z), \alpha z\right\rangle \\
= & \left\langle K^{\prime}(x), \alpha z\right\rangle+\left\langle K^{\prime \prime}(x)(t w), \alpha z\right\rangle+5 \varepsilon|t|^{2}+\frac{1}{2} \alpha^{2}\left\langle K^{\prime \prime}(x)(z), z\right\rangle .
\end{aligned}
$$

Now since $\alpha=\sqrt{\varepsilon}|t|$ we have

$$
\left\langle\underline{K}^{\prime}(x+t w)-K^{\prime}(x)-K^{\prime \prime}(x)(t w), z\right\rangle\left\langle 5 \sqrt{\varepsilon}|t|+\frac{1}{2} \sqrt{\varepsilon}|t|\left\langle K^{\prime \prime}(x)(z), z\right\rangle\right.
$$

which is equivalent to

$$
\left\|\underline{K}^{\prime}(x+t w)-K^{\prime}(x)-K^{\prime \prime}(x)(t w)\right\|<\left(5+\frac{1}{2}\left\|K^{\prime \prime}(x)\right\|\right) \sqrt{\varepsilon}|t|=o|t| .
$$

This completes the proof of (2).

We now proceed with the rest of the proof of Theorem 2. Observe that whenever $K$ is differentiable, Lemma 4 implies that

$$
K^{\prime}(x)=2 C\|x\| x^{*}-2\|x-P(x)\|(x-P(x))^{*} .
$$

In the case where $P(y)$ is multivalued we may take $\underline{P}(y) \in P(y)$. Then it is easy to show that $\underline{K}^{\prime}(y)$ defined by

$$
\underline{K}^{\prime}(y)=2 C\|y\| y^{*}-2\|y-\underline{P}(y)\|(y-\underline{P}(y))^{*}
$$


is a subdifferential of $K$ at $y$. Now let

$$
D(x)=2\|x\| x^{*}=\left(\|x\|^{2}\right)^{\prime} .
$$

By hypothesis $D^{\prime}(x)$ exists and is invertible for all $x$ in $B$. Then by (1) and (4) we have a.e. in $B$,

$$
P(x)=x-D^{-1}\left(2 C\|x\| x^{*}-K^{\prime}(x)\right) .
$$

We also have for any $\underline{P}(y) \in P(y)$,

$$
\underline{P}(y)=y-D^{-1}\left(2\|y\| y^{*}-\underline{K}^{\prime}(y)\right) .
$$

Then from the last two equalities, equation (2) and the assumption on $D$ we obtain that $P^{\prime}(x)$ exists a.e. in $B$ in the sense that

$$
\left\|\underline{P}(y)-P(x)-P^{\prime}(x)(y-x)\right\|=o\|y-x\| \text { a.e. }
$$

\section{REFERENCES}

1. A. D. Alexandrov, Almost everywhere existence of the second differential and some properties of convex surfaces connected with it, Leningrad State Univ. Ann. Math. Ser. 6 (1939), 3-35. MR 2, 155.

2. E. Asplund, Differentiability of the metric projection in finite dimensional Euclidean space, Proc. Amer. Math. Soc. 38 (1973), 218-219.

3. H. Busemann, Convex surfaces, Interscience Tracts in Pure and Applied Mathematics, no. 6, Interscience, New York, 1958.

4. H. Federer, Geometric measure theory, Die Grundlehren der math. Wissenschaften, Band 153, Springer-Verlag, Berlin and New York, 1969.

5. S. P. Fitzpatrick, Personal communication.

Department of Mathematics, Iowa State University, Ames, Iowa 50011 